\title{
Micromechanical model for fatigue limit of metal Additive Manufacturing parts and materials
}

\author{
Anssi Laukkanen, Tom Andersson, Matti Lindroos, Tatu Pinomaa \\ VTT Technical Research Centre of Finland, Kivimiehentie 3, 02240 Espoo, Finland
}

Fatigue remains a challenge especially for high end metal AM parts and materials. From a design perspective, the fatigue limit of an AM solution can be improved upon by optimizing the manufacturing process for a certain material and part. In addition, improved accuracy of design methodologies aids in capturing the features critical for fatigue and quantifies their significance to desired part lifetime as well as providing a basis for their avoidance. In current work we present an overall concept merging thermomechanical process and powder bed solidification modeling to micromechanical analysis of fatigue of the resulting material microstructure. Material features critical to fatigue, particularly surface roughness, internal defects such as porosity and cracks and on the other hand inclusions, can be assessed directly on the basis of AM part microstructure with respect to the resulting fatigue limit. Case analyses consist of maraging steel and nickel alloys. The overall scheme provides a basis for optimization of metal AM solutions against fatigue and multiscale modeling founded basis for fatigue design.

Keywords

Additive Manufacturing, 3D printing

\section{Introduction}

Fatigue of metal additive manufacturing (AM) parts remains a challenge both with respect to better understanding and controlling their appearance as well as evaluating significance to part functionality. In general, two options are available for improving fatigue design of AM components: i) product manufacturing process optimization to minimize e.g. defects responsible for fatigue and ii) increased accuracy of analysis means to better capture those material features critical for fatigue life. Regarding the first option, links to simulation tools for the manufacturing process are considered to create a simulation workflow for minimizing defects in the AM product. Second, micromechanical modeling is employed to capture material features critical for fatigue, particularly surface roughness, internal defects such as porosity and on the other hand inclusions in material microstructure. Use cases utilizing a Ti-6-4 alloy as well as a martensitic microstructure as a basis are utilized, demonstrating how a microstructure based micromechanical assessment procedure can be laid out to quantify the significance of defects in metal AM parts to their fatigue lifetime. The presented tools provide both capabilities towards avoiding fatigue critical features in AM solutions as well as evaluating the implications to product lifetime.

\section{Methods}

The modeling work exploits a phase field model presented in [1] to generate solidification microstructures, different initial configurations and assumptions regarding the alloy and the metal AM processing cycle are utilized to yield different microstructures. The phase field modeling is driven by the thermal output from a metal AM process model, presented in [2]. The microstructural modeling of cyclic damage is based on micromechanics, i.e. crystal plasticity is utilized to extract the deformation response (see [3, 4]), and fatigue performance indicators to quantify and obtain predictions on product lifetime [5]. An example of phase field (PF) generated microstructure is presented in Figure 1. The PF results serve as a direct input for crystal plasticity modeling and subsequent micromechanical modeling of cyclic deformation behavior, and followingly, the fatigue analysis is carried out following the methodologies presented in more detail in [5]. 


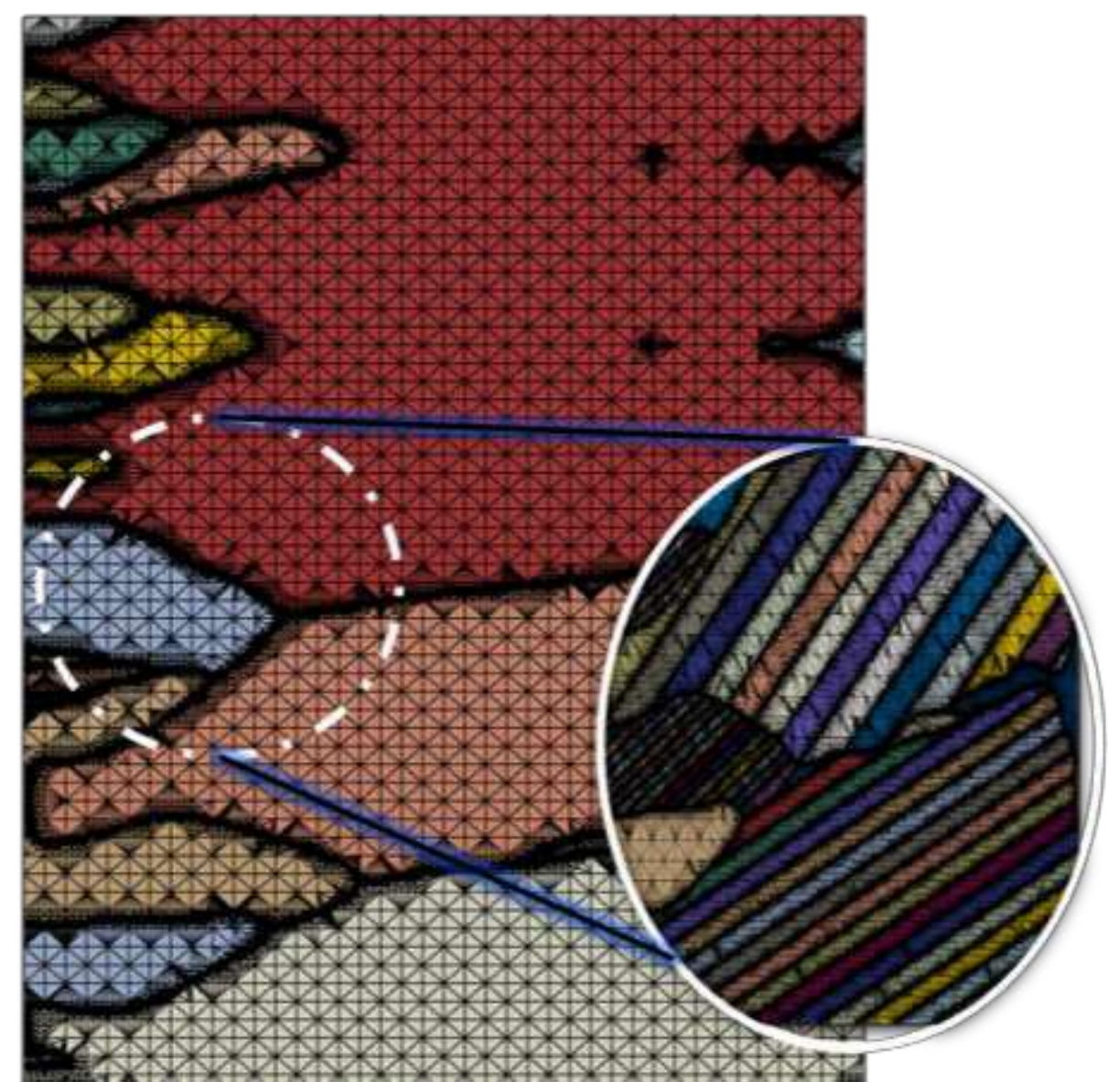

Figure 1: Finite element microstructural model of a phase field solidified Ti-6-4 microstructure, prior microstructure and detail of martensite laths.

\section{Results and discussion}

Following generation of the microstructural models mechanical behavior of different microstructures is evaluated computationally, the results presented in Figure 2. Coarser columnar microstructures are found to be of smaller strength, while greater refinement and density microstructures yield somewhat greater quasistatic mechanical strength also in part due to orientation anisotropies. These results provide a baseline for establishing the deformation behavior of the microstructures of interest. A different type of metallic martensitic microstructure, reminiscent of $\mathrm{H} 13$ tool steel, was assessed with respect to cyclic bulk deformation behavior of a free surface to produce conditions prevalent to fatigue crack nucleation from a surface position. The deformed microstructure is presented in Figure 3 along with contours of cumulative plastic slip. The results indicate how favourably oriented (with respect to applied lateral cyclic deformation) microstructural features nucleate plastic slip near the free surface and are subsequently susceptible to initiation of short microstructure scale fatigue cracks. The finite strain effects on the surface arising from the anisotropic crystal plasticity based material deformation can be clearly observed. 


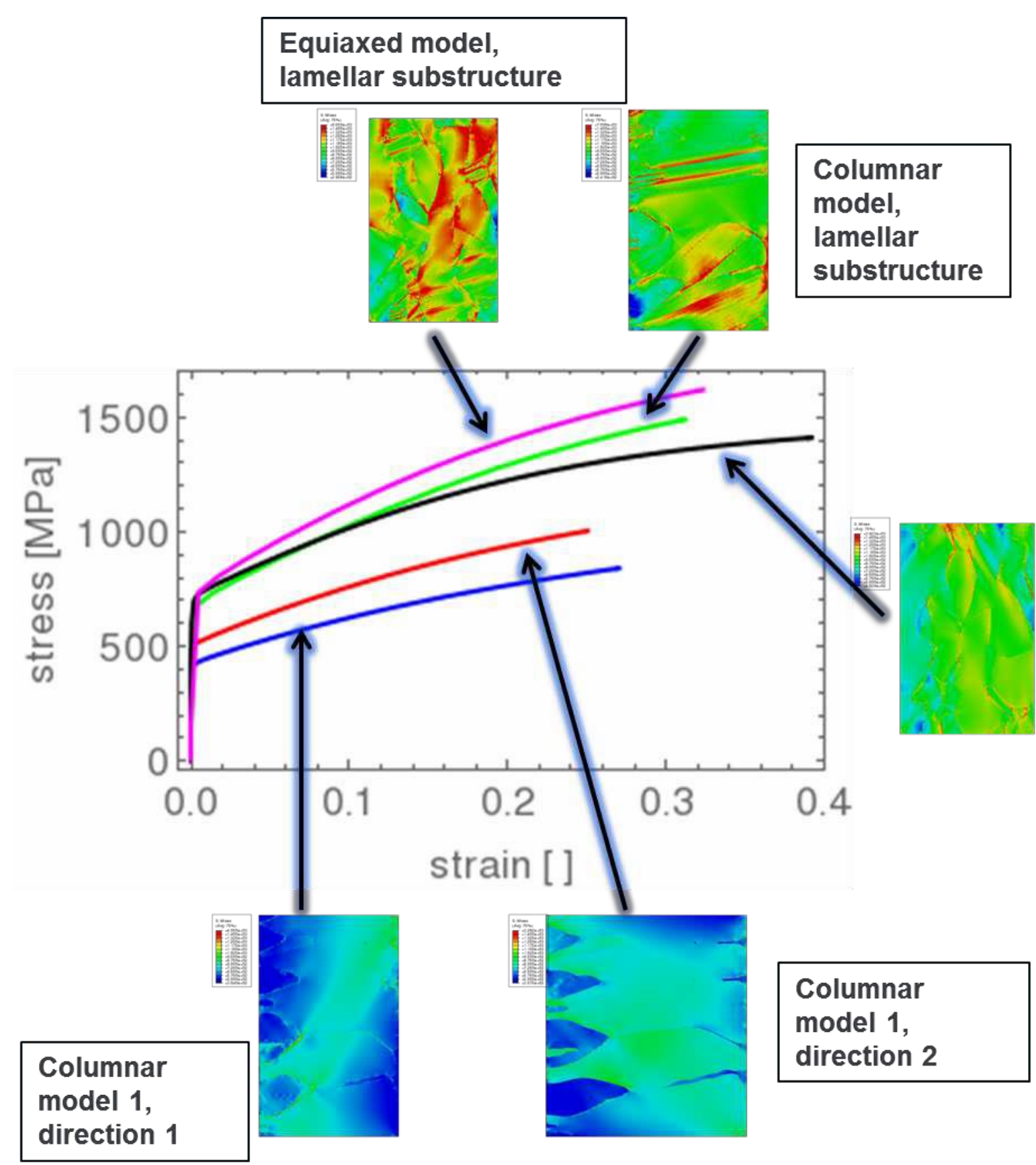

Figure 2: Crystal plasticity simulated true stress-strain curves of different Ti-6-4 microstructures.

The influence of microstructural defects was assessed by introducing small microstructure scale pores and cracks to the microstructural models, the results are presented in Figure 4. The first microstructure contains a smaller aspect ratio pore, while the second one a greater aspect ratio defect. The immediate influence under cyclic deformation is witnessed, the pore inducing a strong discontinuity in terms of the stress-strain field, while the influence of the crack is of even greater significance due to simply its geometric character. The defect sizes are such that they of similar scale as the smallest microstructural hierarchies, and as such, the defects would be of typical size as far as metal AM microstructures are concerned.

The findings for different defect types are summarized in Figure 5 utilizing the fatigue performance indicator approach of [5]. Fatigue crack nucleation is considered for different strain amplitudes and for different microstructures, with and without defects. The differences in coarser vs more refined microstructures can be identified and especially the deleterious effects of microstructure scale defects. 
Porosity and especially the microstructural scale cracks are seen to decrease the cycles to short crack nucleation by a factor of $2-5$, the difference as such being very significant. The baseline fatigue life is established by utilizing bulk microstructures without defects as a reference against which the other microstructural models and their predictions are compared to.

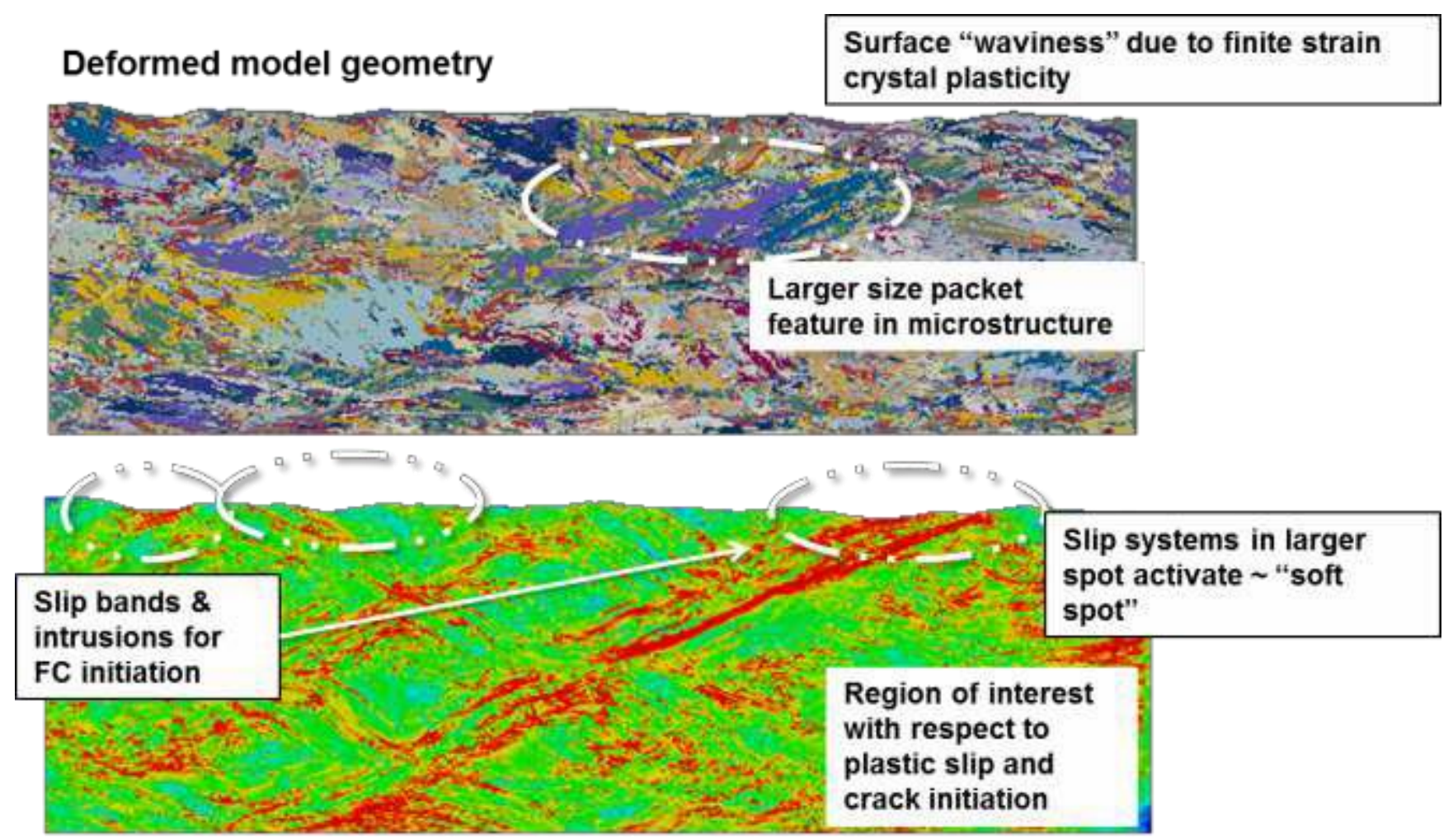

Figure 3: Deformed microstructure (top) and cumulative plastic slip of a free surface (bottom) following cyclic loading of a martensitic microstructure.

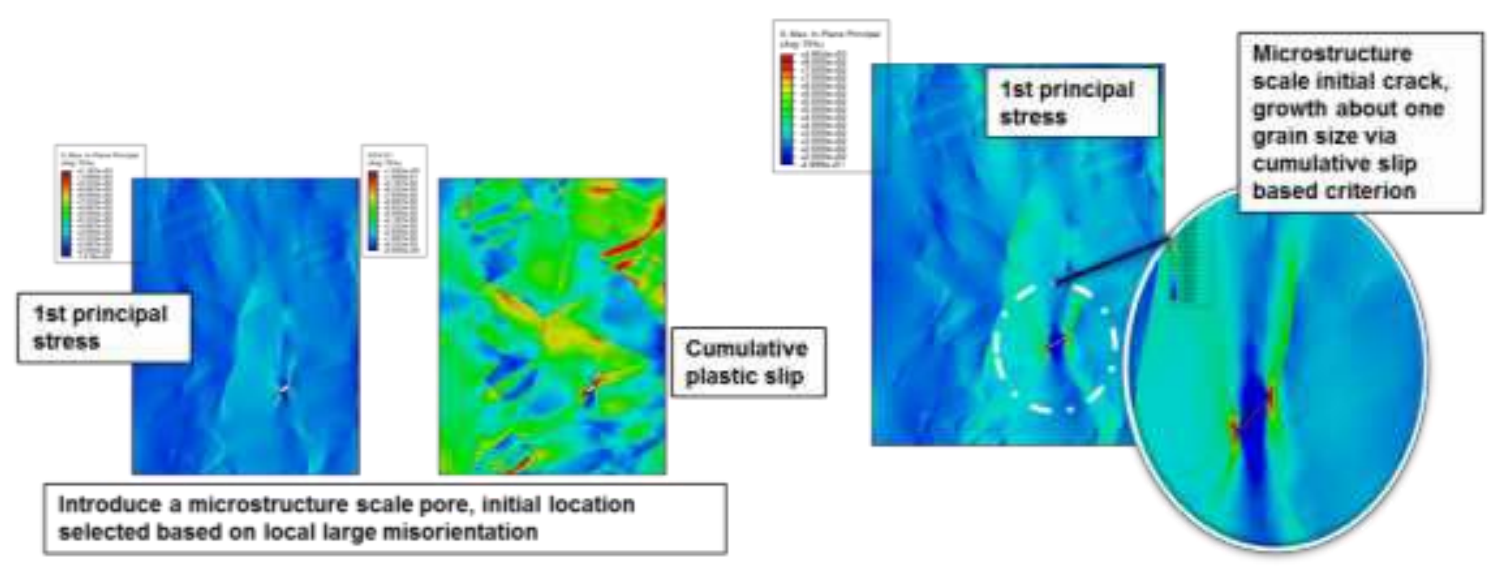

Figure 4: Influence of microstructural defects: small pore (left) and crack like defect (right). Contours of $1^{\text {st }}$ principal stress and cumulative plastic slip. 


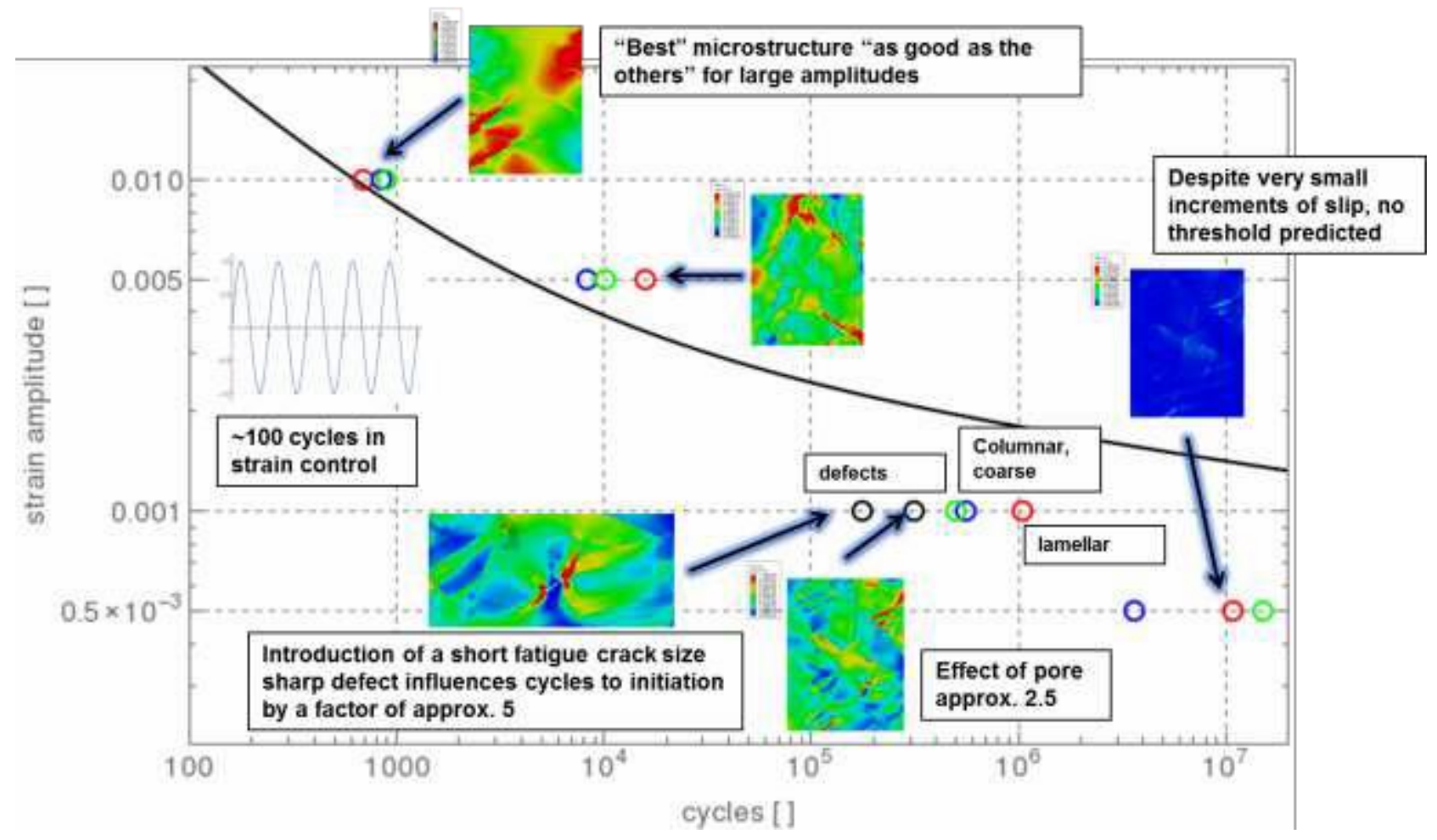

Figure 5: Summary of predictions for cycles to short fatigue crack nucleation.

\section{Summary and Conclusions}

A micromechanical approach was adopted to predict based on crystal plasticity modeling the cycles required for nucleation of fatigue cracks in metal AM microstructures. The findings can be concluded as follows:

- The linkage of phase field and thermomechanical process modeling was successfully utilized to create trial microstructures for modeling fatigue damage, establishing a design chain from the metal AM process as the way to product and material solution performance.

- The micromechanical approach is able to capture features considered relevant for fatigue damage nucleation from part surface due to cyclic loading.

- The significance of microstructure scale defects and discontinuities was overall emphasized as expected, porosity and cracks appearing clearly more hazardous over behavior of bulk microstructure. Similar arguments can be made for inclusions.

- The methodologies provide a basis for performing microstructure based evaluation of fatigue damage and lifetime, enabling quantification of significance of specific defects and improved accuracy of fatigue analysis and design.

- The utilization of process and solidification models simultaneously to drive material performance models provides a basis for development of integrated computational materials engineering solutions for metal additive manufacturing, enabling an extremely rich design space with respect to fatigue also encompassing material and powder alloying and processing.

\section{References}

[1]: Pinomaa, T., Andersson, T., Gurevich, T., Lindroos, T., Laukkanen, A., Laitinen, T., Provatas, N. 2018. Phase field modeling of selective laser melting. WorldPM2018. 
[2] : Laakso, P., Riipinen, T., Laukkanen, A., Andersson, T., Jokinen, A., Revuelta, A., Ruusuvuori, K. 2016. Optimization and simulation of SLM process for high density $\mathrm{H} 13$ tool steel parts. Physics Procedia, 83, pp. 26-35.

[3] : Lindroos, M., Laukkanen, A., Cailletaud, G., Kuokkala, V-T., 2018. Microstructure based modeling of the strain rate history effect in wear resistant Hadfield steels. Wear, 396-397, pp. 56-66.

[4] : Lindroos, M., Laukkanen, A., Kuokkala, V-T., 2017. A crystal plasticity approach for shear banding in hot rolled high-strength steels. Metallurgical and Material Transactions A, 48, pp. 5608-5615.

[5] : Laukkanen, A., Lindroos, M., Andersson, T., Verho, T., Pinomaa, T., 2017. Micromechanical modeling of failure behavior of metallic materials. Rakenteiden Mekaniikka, 50, 271-274.

[6] I. Gibson, D.W. Rosen, B. Stucker, Additive Manufacturing Technologies: 3D Printing, Rapid Prototyping, and Direct Digital Manufacturing, Springer (2015), pp. 1-498. 